\title{
Spinal Cord Injury Immediately Changes the State of the Brain
}

\author{
Juan Aguilar, ${ }^{1}$ Desiré Humanes-Valera, ${ }^{1}$ Elena Alonso-Calviño, ${ }^{1}$ Josué G. Yague, ${ }^{1}$ Karen A. Moxon, ${ }^{2}$ Antonio Oliviero, ${ }^{1}$ \\ and Guglielmo Foffani ${ }^{1,2}$ \\ ${ }^{1}$ Hospital Nacional de Parapléjicos, Servicio de Salud de Castilla-La Mancha, 45071 Toledo, Spain, and ${ }^{2}$ School of Biomedical Engineering, Science, and \\ Health Systems, Drexel University, Philadelphia, Pennsylvania 19104
}

\begin{abstract}
Spinal cord injury can produce extensive long-term reorganization of the cerebral cortex. Little is known, however, about the sequence of cortical events starting immediately after the lesion. Here we show that a complete thoracic transection of the spinal cord produces immediate functional reorganization in the primary somatosensory cortex of anesthetized rats. Besides the obvious loss of cortical responses to hindpaw stimuli (below the level of the lesion), cortical responses evoked by forepaw stimuli (above the level of the lesion) markedly increase. Importantly, these increased responses correlate with a slower and overall more silent cortical spontaneous activity, representing a switch to a network state of slow-wave activity similar to that observed during slow-wave sleep. The same immediate cortical changes are observed after reversible pharmacological block of spinal cord conduction, but not after sham. We conclude that the deafferentation due to spinal cord injury can immediately (within minutes) change the state of large cortical networks, and that this state change plays a critical role in the early cortical reorganization after spinal cord injury.
\end{abstract}

\section{Introduction}

Spinal cord injury produces abrupt irreversible deafferentation of cortical circuits. This deafferentation can lead to major long-term reorganization of cortical topographic maps, reflecting remarkable plasticity in the adult brain (Wall and Egger, 1971; Jain et al., 1997, 2008; Bruehlmeier et al., 1998; Green et al., 1998; Curt et al., 2002; Endo et al., 2007; Ghosh et al., 2009, 2010; Tandon et al., 2009). Cortical reorganization plays a critical role for functional recovery (Kaas et al., 2008; Ghosh et al., 2009; Kao et al., 2009), but excessive or aberrant reorganization can have pathological consequences, such as phantom sensations (Moore et al., 2000) and neuropathic pain (Wrigley et al., 2009). To understand the pathophysiological mechanisms of long-term cortical reorganization - and to develop timely interventions to manage that reorganization-it is necessary to investigate the changes that occur immediately after the lesion. Even though a vast literature exists about the immediate cortical changes occurring after peripheral injuries (Merzenich et al., 1983; Kelahan and Doetsch, 1984; Wall and Cusick, 1984; Calford and Tweedale, 1988, 1991a,b; Cusick et al., 1990; Turnbull and Rasmusson, 1990; Byrne and Calford, 1991; Doetsch et al., 1996; Faggin et al., 1997), little is known about the immediate cortical changes induced by spinal cord injury.

This work was supported by Fundación para la Investigación Sanitaria en Castilla-La Mancha, Fondo de Investigacion Sanitaria del Instituto de Salud Carlos III Grant PI08/1852 PI08/1810 (Spain), and National Institutes of Health Grant R01 NS057419. We thank Dr. Antonio Canedo and Dr. Casto Rivadulla for useful comments on the manuscript.

Correspondence should be addressed to either Juan Aguilar or Guglielmo Foffani, Hospital Nacional de Parapléjicos, Servicio de Salud de Castilla-La Mancha, Finca La Peraleda s/n, 45071 Toledo, Spain, E-mail: jdaguilar@sescam.jccm.es or guglielmo.foffani@drexel.edu.

DOI:10.1523/JNEUROSCI.0379-10.2010

Copyright $\odot 2010$ the authors $\quad 0270-6474 / 10 / 307528-10 \$ 15.00 / 0$
To address this issue, we investigated acute neurophysiological changes occurring immediately (i.e., within $1 \mathrm{~h}$ ) after spinal cord injury in the primary somatosensory cortex of anesthetized rats. To clearly separate deafferented and intact cortical areas, we used a thoracic complete transection model (level T9-T10) that has been shown to induce long-term cortical reorganization in rats (Endo et al., 2007). This lesion blocks all ascending inputs from the hindpaws, but does not affect inputs from the forepaws. We simultaneously recorded local field potentials (LFPs) and multiunit activity from the hindpaw representation (deafferented) and the forepaw representation (intact) of the primary somatosensory cortex. We monitored the cortical responses evoked by somatosensory stimuli and the cortical spontaneous activity before and immediately after the spinal cord transection.

\section{Materials and Methods}

Experiments were performed following the rules of International Council for Laboratory Animal Science, European Union regulation 86/609/ EEC, and were approved by the Ethical Committee for Animal Research of the Hospital Nacional de Parapléjicos (Toledo, Spain). A total of 31 male Wistar rats were used in this study, divided into three groups: (1) 14 animals with spinal cord transection; (2) 8 animals with pharmacological block of the spinal cord; and (3) 9 animals with "sham."

\section{Experimental protocol}

The main experimental protocol was performed in the group of animals that received spinal cord transection $(n=14)$. Animals were anesthetized with urethane $(1.5 \mathrm{~g} / \mathrm{kg}$, i.p.). The body temperature of the animal was kept constant $\left(36.5^{\circ} \mathrm{C}\right)$ using an automatically controlled heating pad. A laminectomy was performed at thoracic level (T9-T10) keeping the dura mater intact and covered with agar $4 \%$ to protect the area. The animals were placed in a stereotaxic frame (SR-6 Narishige Scientific Instruments). Lidocaine $2 \%$ was applied over the body surface in contact with the frame and over the areas for incisions. The skin of the head was softly 
removed and the skull was exposed. A craniotomy was performed on the right side of the midline over the somatosensory cortex [AP: 1 to -4 ; ML: 1-5; atlas of Paxinos and Watson (1986)], and the cisterna magna was opened to guarantee the stability of the recordings. Small incisions in the dura mater were performed to allow the recording electrodes to be lowered into the cerebral cortex. Once the electrodes were placed in the hindpaw and forepaw representations of the primary somatosensory cortex, we performed the prelesion protocol, recording evoked responses and spontaneous activity with intact spinal cord. We then performed the complete transection of the spinal cord with a scalpel blade. Immediately after transection, a few pulses of electrical stimulation of the hindpaw at very high intensity $(10 \mathrm{~mA})$ were applied to confirm that no physiological responses were evoked in the cortex by stimuli delivered below the level of the lesion. The complete cut of the spinal cord was visually confirmed under the surgical microscope by the total separation of the borders. Electrophysiological recordings were continuously acquired during the transection to confirm the stability of the recordings from control conditions to after lesion. Between 10 and $30 \mathrm{~min}$ after the transection, we started the postlesion protocol, recording evoked responses and spontaneous activity with the spinal cord transected. Based on the absence of reflexes to forepaw stimuli and on the absence of corneal reflex, animals never received additional anesthesia between the prelesion protocol and the postlesion protocol. We never observed spontaneous whisker movements during the recordings.

\section{Electrophysiology}

Electrophysiological extracellular recordings techniques were similar to our previous works (Aguilar et al., 2008; Foffani et al., 2009). Recordings were obtained using tungsten electrodes with 4-5 M $\Omega$ impedance at $1000 \mathrm{~Hz}$ (TM31C40KT and TM31A50KT of WPI). Two electrodes were lowered in the infragranular somatosensory cortex, one in the forepaw area (anteroposterior: $0-0.5 \mathrm{~mm}$; mediolateral: $3.5-4.5 \mathrm{~mm}$ ) and the other one in the hindpaw area (anteroposterior: -0.5 to $-1 \mathrm{~mm}$; mediolateral: 2-2.5 mm), following the coordinates of Chapin and Lin (1984). Beside the stereotactic coordinates, the exact anteroposterior and mediolateral locations of the electrodes were adjusted with multiple penetrations (up to five, typically three penetrations per electrode) to optimize the physiological responses to the corresponding peripheral stimuli (maximum amplitude, minimal latency). To adjust the depth, we first identified layer 4, where response latencies are the shortest, and then lowered the electrodes to the infragranular layers (depth: 1.1-1.8 mm). Once the final locations were defined, the electrodes were fixed in the place and were not moved throughout the entire experiment. We specifically targeted the infragranular layers because they are (1) the cortical layers expressing maximal convergence of excitatory and inhibitory inputs, both local and long-range; (2) the main origin of cortical outputs; and (3) the layers where the majority of active states originate (CastroAlamancos, 2000; Sanchez-Vives and McCormick, 2000; CastroAlamancos and Rigas, 2002; Sakata and Harris, 2009). The infragranular cortex is thus particularly appropriate to investigate at the network level cortical changes after deafferentation. Nonetheless, we would like to note that because active states - both spontaneous and evoked-rapidly synchronize all cortical layers, the results of the present study are highly likely to extend beyond the infragranular cortex.

All recordings were preamplified, filtered ( $1 \mathrm{~Hz}$ to $3 \mathrm{kHz}$ ), and amplified using a modular system (Neurolog; Digitimer). Analog signals were converted into digital data at $20 \mathrm{kHz}$ sampling rate and 16 bit quantization using a CED power 1401 (Cambridge Electronics Design) controlled by Spike2 software (v6 Cambridge Electronics Design). Signals were stored on a hard disk of a PC for posterior analysis.

\section{Peripheral stimulation}

Electrical pulses were applied using bipolar needle electrodes located subcutaneously in the wrist of the forepaw and of the hindpaw, one pole in each side of the paw. The rationale for this stimulation was to activate all types of somatosensory fibers originating within the paws, including tactile, proprioceptive, and nociceptive fibers. The protocol consisted of a total of 100 pulse stimuli with duration of $1 \mathrm{~ms}$ and frequency of $0.5 \mathrm{~Hz}$. Two different intensities were applied: low intensity $(0.5 \mathrm{~mA})$ and high intensity $(5 \mathrm{~mA})$. Low-intensity stimuli were intended to activate only a fraction of the available fibers, mainly low-threshold primary fibers running through the lemniscal pathway, from the dorsal columns to the brainstem (Lilja et al., 2006). High-intensity stimuli were intended to activate the maximum number of fibers, including high-threshold primary fibers that make synapse in the dorsal horns of the spinal cord, in turn activating the spinothalamic tract (Lilja et al., 2006).

\section{Control experiments}

In a number of animals $(n=8)$, blockers of voltage-dependent sodium channels (TTX or lidocaine) were applied topically in the spinal cord at thoracic level (T9-T10). With this treatment it was possible to avoid the traumatic damage of the spinal cord and reproduce the same effects of the transection in terms of total deafferentation of lower trunk and hindlimbs. The experimental protocol was the same as previously described for the spinal transection, but in this case a few drops of TTX $(50 \mu \mathrm{M})$ or lidocaine ( $2 \%$ in saline) were applied directly over the spinal cord. Immediately a cotton piece was soaked in the same compound and delicately placed over the spinal cord. The block of axonal conduction was verified by the absence of any physiological response to a few pulses of electrical stimulation delivered to the hindpaw at very high intensity ( 10 $\mathrm{mA}$ ). As soon as the effect of the blocked axonal conduction was observed, we performed our protocol to record cortical evoked responses and spontaneous activity. Immediately after, the cotton piece soaked with TTX or lidocaine was removed and the area was rinsed with saline, every 10 min during a total of $1-2 \mathrm{~h}$, until the complete recovery of the cortical evoked responses to hindpaw stimuli.

We also performed a set of "sham" experiments $(n=9)$ in which the spinal cord remained intact after the laminectomy for the entire duration of the experiment. Besides the absence of spinal cord lesion, the experimental protocol was the same as in the transected animals.

\section{Data analysis}

Cortical responses. LFP responses were obtained by averaging across stimuli the raw signals recorded from the electrodes. LFP response amplitude was evaluated as the absolute value of the negative peak in the average response. Repeating the analyses using a peak-to-peak measure yielded essentially the same results, with slightly greater amplitudes (data not shown). Multiunit responses were obtained by (1) bandpass filtering the raw signals at high frequencies $(800-3000 \mathrm{~Hz}),(2)$ detecting all spikes that exceeded the background noise by at least 5 SDs, and (3) averaging the spikes across stimuli to construct peristimulus time histograms (PSTHs). The magnitude of multiunit responses was evaluated as the average number of spikes per stimulus emitted in the first $30 \mathrm{~ms}$ after the stimulus.

Spontaneous activity. Cortical spontaneous activity was studied in recordings at least $200 \mathrm{~s}$ long, performed between the low-intensity stimulation and the high-intensity stimulation. To quantitatively evaluate the level of cortical spontaneous activity, we extracted the rectified multiunit activity (rMUA) by bandpass filtering the raw signals at high frequencies and rectifying the resulting signal. Note that the multiunit signal we used above to construct the PSTHs of the responses is a point process, whereas the rMUA used here is a time series, representing the compound spiking activity of the local population of neurons around the electrode. The rMUA was chosen because it correlates well with the membrane potential of intracellular recordings and it is thus a good measure of the level of activity in cortical networks (Hasenstaub et al., 2007). The rMUA spectrum was estimated by (1) subtracting the mean to the rMUA, (2) calculating its autocorrelation function, and (3) estimating the power spectral density with Thomson multitaper method. The peak frequency of the rMUA spectrum was used as a measure of the average alternation rate between active and silent states. The neurophysiological consistency of this measure was visually verified in all recordings, and recordings in which the rMUA did not reach a sufficient signal-to-noise ratio to properly estimate the spectrum were excluded from the analyses. We also calculated the mean of the rMUA (in the time domain) as a measure of the overall level of cortical spontaneous activity.

Correlation between cortical spontaneous activity and evoked responses. We investigated the relation between cortical spontaneous activity and 
Before spinal transection
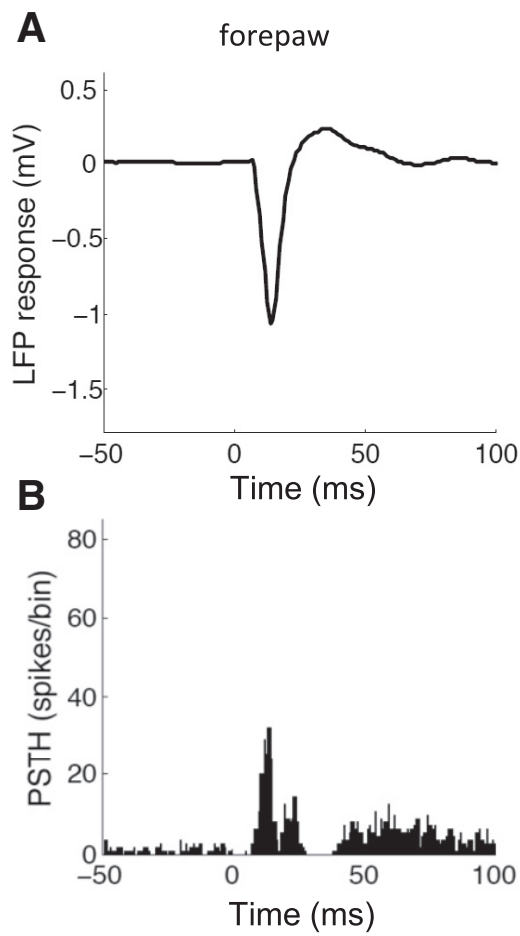

hindpaw
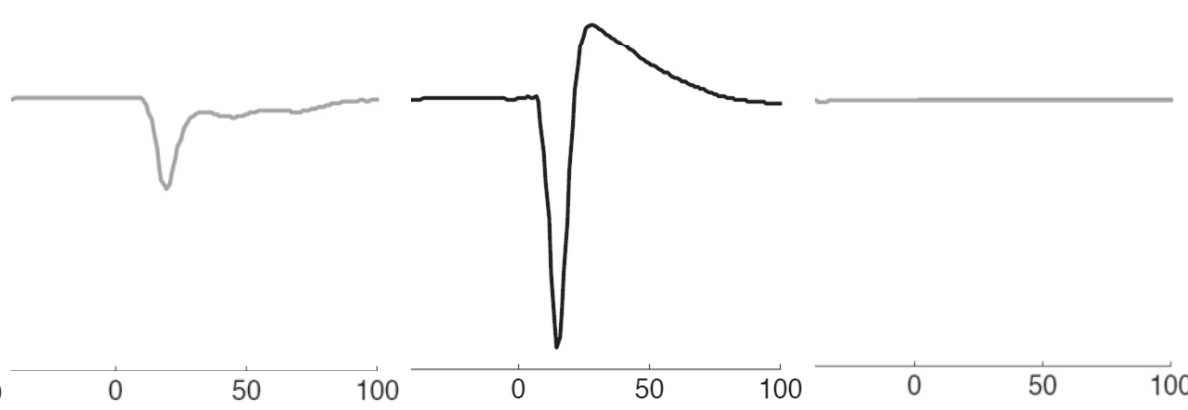

After spinal transection
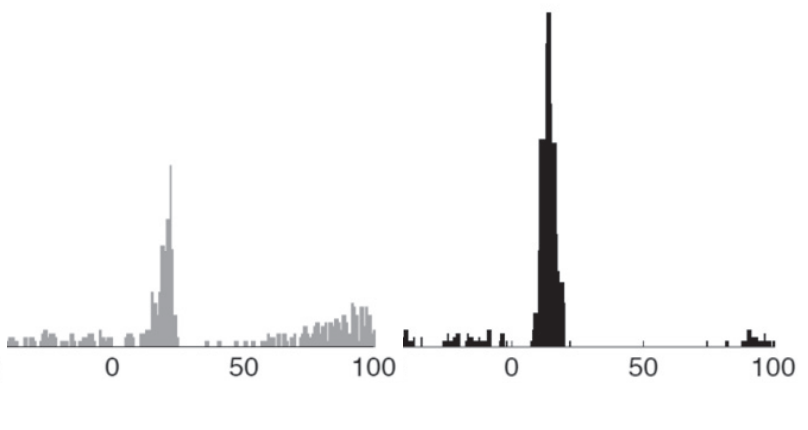

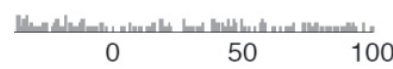

Figure 1. Changes of cortical responses. $\boldsymbol{A}$, LFP responses evoked in the forepaw cortex by forepaw stimuli (black) and in the hindpaw cortex by hindpaw stimuli (gray) before (left) and immediately after (right) complete thoracic transection of the spinal cord in a representative animal. $\boldsymbol{B}$, PSTHs representing multiunit responses recorded from the same electrodes. LFP responses and PSTHs were evaluated from 100 stimuli. Time 0 ( $x$-axis) represents stimulus onset. Spinal cord transection completely abolished the responses evoked by hindpaw stimuli, as expected, but remarkably enhanced the responses evoked by forepaw stimuli.

Table 1. Cortical responses before and immediately after complete thoracic transection of the spinal cord, pharmacological block, or sham

\begin{tabular}{|c|c|c|c|c|}
\hline & \multicolumn{2}{|l|}{ Before } & \multicolumn{2}{|l|}{ After } \\
\hline & HP & FP & $\mathrm{HP}$ & $\mathrm{FP}$ \\
\hline \multicolumn{5}{|c|}{ Spinal cord transection $(n=14)$} \\
\hline Low intensity & $0.27 \pm 0.12$ & $0.30 \pm 0.16$ & - & $0.31 \pm 0.20$ \\
\hline \multicolumn{5}{|c|}{ Spinal cord pharmacological block $(n=8)$} \\
\hline High intensity & $0.45 \pm 0.32$ & $1.08 \pm 0.57$ & - & $1.52 \pm 0.83$ \\
\hline Low intensity & $0.17 \pm 0.07$ & $0.23 \pm 0.14$ & - & $0.26 \pm 0.21$ \\
\hline Low intensity & $0.19 \pm 0.13$ & $0.23 \pm 0.15$ & $0.16 \pm 0.10$ & $0.21 \pm 0.15$ \\
\hline
\end{tabular}

Data are amplitudes of LFP responses (in millivolts) to high- or low-intensity stimuli. FP, Forepaw; HP, hindpaw.

evoked responses by evaluating, on a stimulus-by-stimulus basis, the median of the rMUA in a $100 \mathrm{~ms}$ prestimulus window and the single-trial LFP amplitude in the $100 \mathrm{~ms}$ poststimulus window (see Fig. 3B). In each animal and condition, single-trial LFP amplitudes were sorted based on the prestimulus rMUA median, averaged over blocks of 10 stimuli to obtain 10 points per animal, and finally averaged across animals.

\section{Statistical analyses}

Changes in cortical responses were evaluated with two-way repeatedmeasures ANOVAs, with prelesion-postlesion as first factor with two levels (before spinal transection and after spinal transection) and stimulus as second factor with two levels (low intensity and high intensity).

Changes in cortical spontaneous activity were evaluated with two-way mixed ANOVAs, with prelesion-postlesion as first factor with two levels of repeated measures (before spinal transection and after spinal transection) and hindpaw-forepaw as second factor with two levels of independent measures (forepaw cortex and hindpaw cortex).

Tukey honestly significant difference test was used for all post hoc comparisons.
Correlations between prestimulus activity and poststimulus responses were performed with Pearson correlation coefficients.

All results were considered significant at $p<0.05$.

\section{Results}

\section{Changes of cortical responses}

We first investigated immediate changes in the cortical LFP responses evoked by electrical stimuli delivered to the hindpaw and forepaw before and after thoracic transection of the spinal cord (Fig. 1A). LFP responses are the intracranial equivalent of the somatosensory evoked potentials used in human studies. In intact animals $(n=14)$, LFP responses in the forepaw cortex to forepaw stimuli displayed greater amplitude than LFP responses in the hindpaw cortex to hindpaw stimuli (Table 1), which is consistent with our previous work (Moxon et al., 2008). Immediately after transection of the spinal cord, LFP responses to hindpaw stimuli-below the level of the lesion-were abolished, 

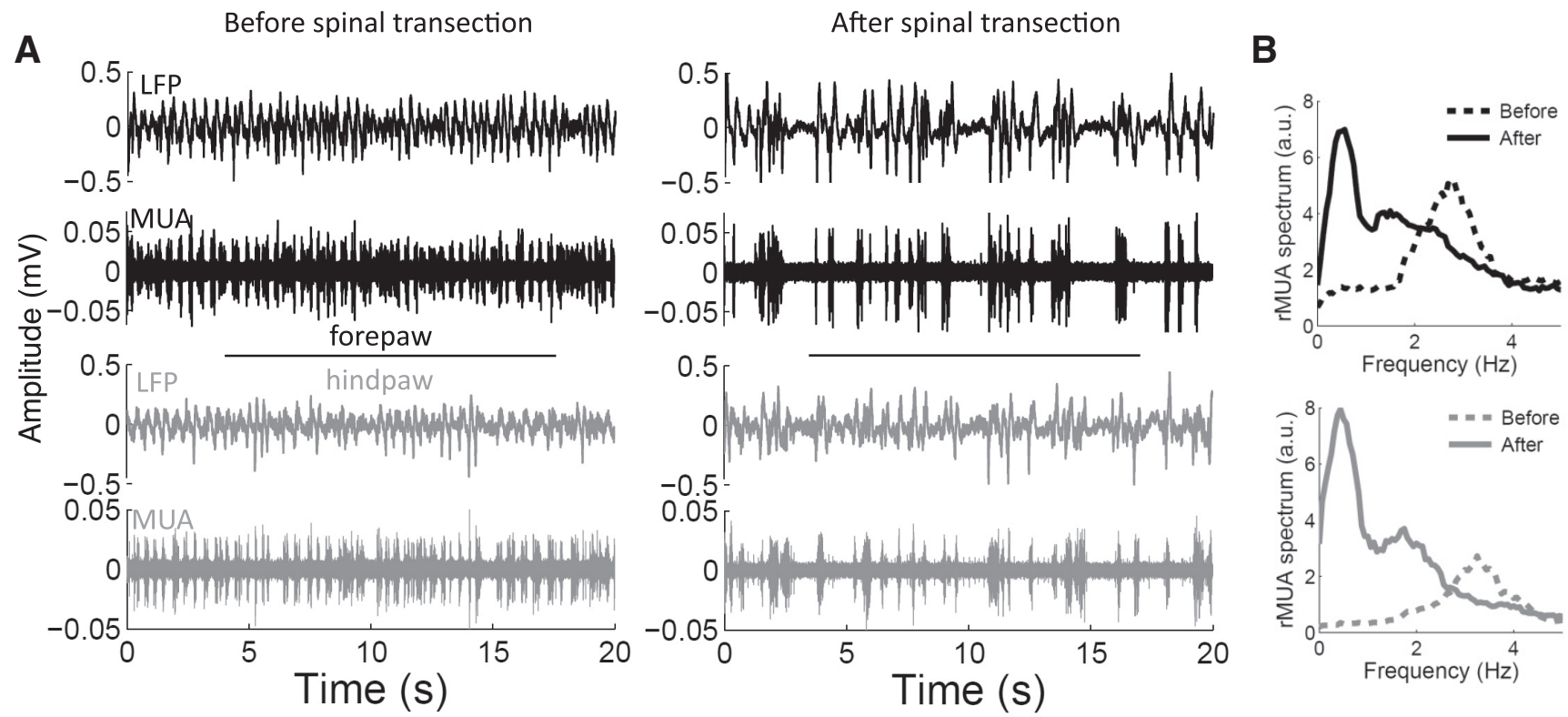

Figure 2. Changes of cortical spontaneous activity. $\boldsymbol{A}$, Examples of 20 s spontaneous recordings of LFPs and MUA from the forepaw cortex (black) and the hindpaw cortex (gray) before (left) and immediately after (right) complete thoracic transection of the spinal cord in a representative animal. $\boldsymbol{B}$, Power spectrum of the rMUA corresponding to the recordings shown in $\boldsymbol{A}$. Before spinal cord transection, the somatosensory cortex of this animal was oscillating at delta frequencies ( $\sim 3 \mathrm{~Hz}$ ), but the oscillation switched to slow-wave activity ( $\sim 0.5 \mathrm{~Hz}$ ) after the spinal cord transection.

confirming the complete spinal cord lesion. Conversely, LFP responses evoked in the forepaw cortex by forepaw stimuli-above the level of the lesion-remarkably increased (Table 1) (two-way ANOVA, prelesion-postlesion factor: $p=0.0001)$. This increased amplitude was specifically observed in response to highintensity ( $5 \mathrm{~mA}$ ) stimuli (interaction factor: $p=0.0004$; Tukey: $p=0.0002)$, but not in response to low-intensity (0.5 mA) stimuli (Tukey: $p=0.99$ ).

These results were then corroborated by evaluating the multiunit responses (Fig. $1 B$ ): similarly to the LFP responses, the magnitude of multiunit responses evoked in the forepaw cortex by forepaw stimuli significantly increased after spinal cord transection (two-way ANOVA, prelesion-postlesion factor: $p=$ 0.0109), specifically in response to high-intensity stimuli (from $5.2 \pm 2.7$ to $7.0 \pm 2.5$ spikes/stimulus; interaction factor: $p=$ 0.000393; Tukey: $p=0.0002$ ), but not in response to lowintensity stimuli ( $1.2 \pm 1.0$ and $1.4 \pm 1.4$ spikes/stimulus; Tukey: $p=0.73)$.

\section{Changes of cortical spontaneous activity}

To get insights into the possible network mechanisms underlying the increased forepaw responses after thoracic transection, we studied the immediate changes of cortical spontaneous activity (Fig. 2). In intact rats, the cortical spontaneous activity consisted of the characteristic behavior induced by urethane anesthesia, in which periods of synchronous network activity (active states) alternate with periods of silence (silent states). This behavior was evident in the MUA, and could be quantified by the peak frequency of the rectified MUA spectrum. The alternation between active and silent states occurred on average at $1.10 \pm 0.86 \mathrm{~Hz}$ in the hindpaw cortex (range $0.37-2.94 \mathrm{~Hz}$ ) and at $1.00 \pm 0.67 \mathrm{~Hz}$ in the forepaw cortex (range $0.37-2.31 \mathrm{~Hz}$ ), with high correlation between the two cortices (Pearson: $r=0.97, p=0.0000002, n=$ 12; in two animals the MUA recorded from the hindpaw cortex had poor signal-to-noise ratio, so these animals were excluded from this analysis). After thoracic transection of the spinal cord, the alternation between active and silent states became slower, occurring on average at $0.50 \pm 0.12 \mathrm{~Hz}$ (range $0.37-0.69 \mathrm{~Hz}$ ) in the hindpaw cortex and at $0.50 \pm 0.10 \mathrm{~Hz}$ (range $0.37-0.69 \mathrm{~Hz}$ ) in the forepaw cortex (two-way ANOVA; prelesion-postlesion factor: $p=0.0024$; hindpaw-forepaw factor: $p=0.75$; interaction: $p=0.77 ; n=12$ ), again with high correlation between the two cortices (Pearson: $r=0.97, p=0.00000008$ ). The slower alternation between active and silent states after thoracic transection was particularly evident when the cortex started from a relatively faster oscillatory state (as in Fig. 2). After the lesion, all animals switched to a cortical state of slow-wave activity similar to that observed during slow-wave sleep $(<1 \mathrm{~Hz})$ (Steriade et al., 1993; Crunelli and Hughes, 2010). This slower alternation between active and silent states after thoracic transection corresponded to a globally more silent cortical spontaneous activity, as evidenced by a decreased mean amplitude of the rectified MUA (two-way ANOVA; prelesion-postlesion factor: $p=0.0031$; hindpaw-forepaw factor: $p=0.42$; interaction: $p=0.13 ; n=12$ ).

\section{Relation between cortical spontaneous activity and evoked responses}

To establish a direct relationship between slower/more silent cortical spontaneous activity and increased forepaw responses, we investigated whether the level of cortical spontaneous activity immediately preceding each forepaw stimulus affected the subsequent single-trial LFP response to the stimulus (Fig. $3 A$ ). In intact rats $(n=14)$, we found a clear inverse correlation between spontaneous activity and forepaw response (Fig. $3 B, C$ ): the greater the median of the rectified MUA immediately preceding the stimulus, the smaller the LFP response evoked by the subsequent stimulus (Pearson: $r<-0.96, p<0.00001$ ) (Fig. $3 C$ ). After spinal transection, this inverse correlation was maintained $(r<-0.93, p<0.0001)$ (Fig. 3C), but the lower values of spontaneous activity (expected from the previous paragraph) were associated with even higher values of response. The slope $(S)$ of this inverse correlation between spontaneous activity (in microvolts) and evoked responses (in millivolts) was much steeper for high-intensity stimuli (before transection, $S=-0.110$; after 
A

LFP

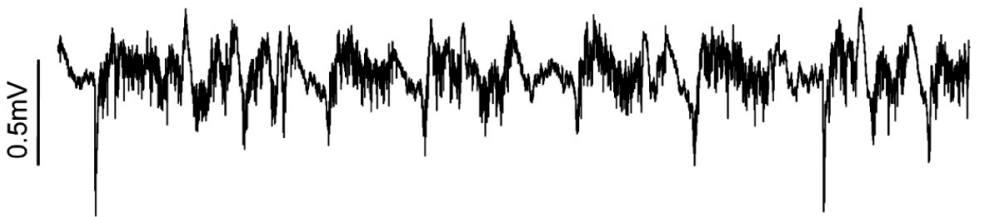

MUA

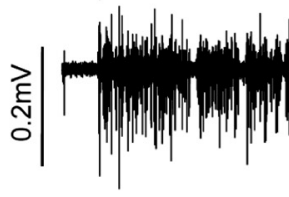

Stimuli

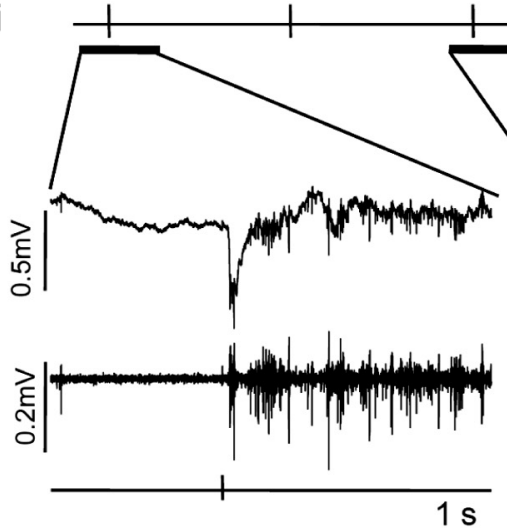

$1 \mathrm{~s}$
B
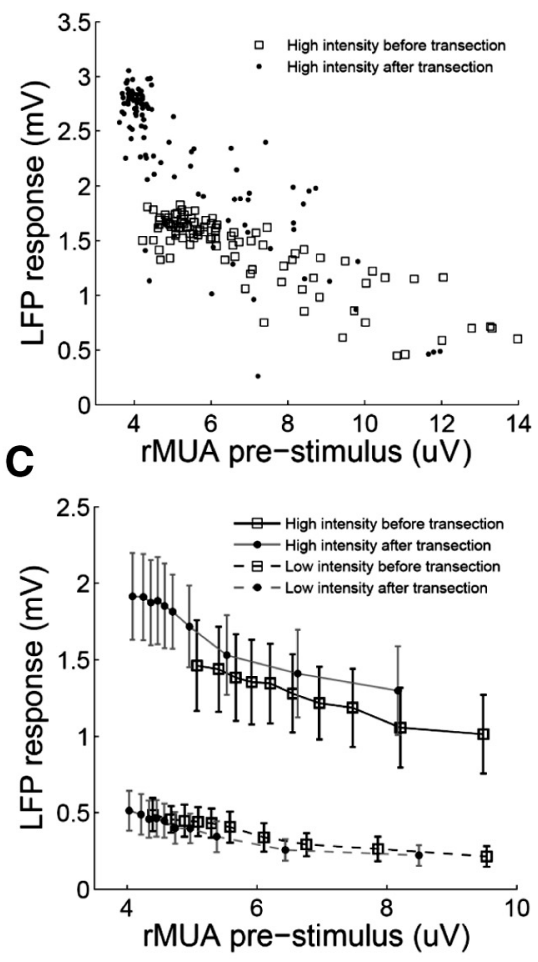

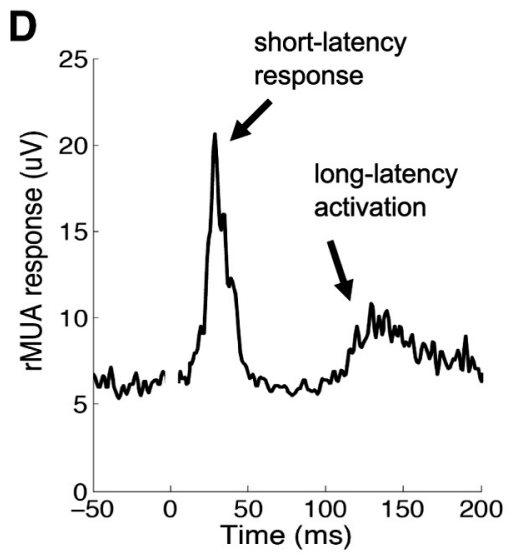

E

After spinal transection

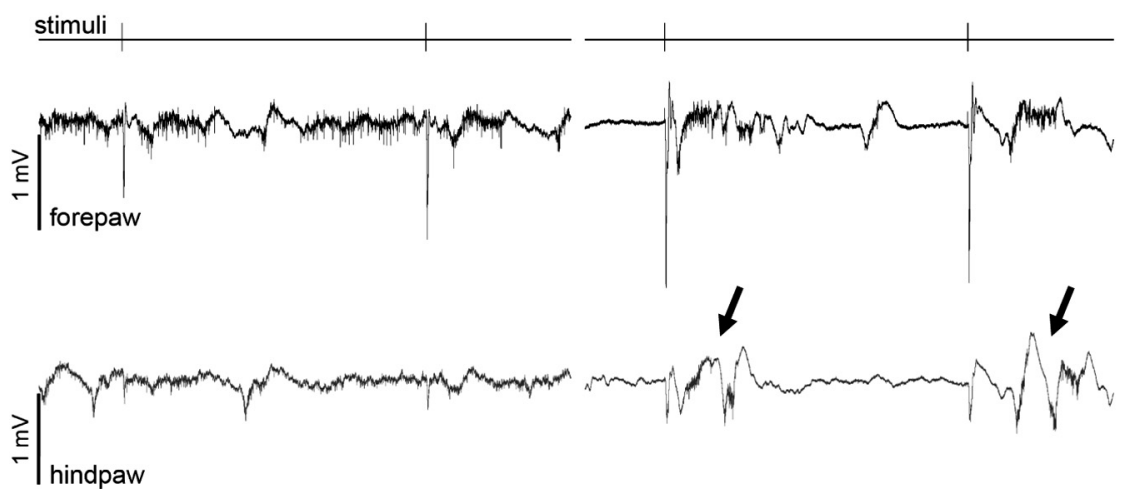

Figure 3. Relation between cortical spontaneous activity and evoked responses. $A$, Example of $10 \mathrm{~s}$ recordings of LFPs and MUA in response to electrical somatosensory stimuli delivered every $2 \mathrm{~s}$. The time around two representative stimuli is expanded on the bottom: if immediately before the stimulus the cortex is silent (small MUA), then the LFP response is large; if immediately before the stimulus the cortex is active (large MUA), then the LFP response is small. $B$, Single-trial data from a representative animal: rMUA before stimulus ( $x$-axis) is plotted against the LFP response ( $y$-axis) for 100 high-intensity stimuli delivered before (empty squares) and after (filled dots) complete thoracic transection of the spinal cord. C, Pooled data from all the animals ( $n=14$ ). For each animal, stimuli were sorted based on the rMUA before stimulus and LFP responses were averaged in blocks of 10 stimuli, to obtain 10 points per animal, which were then averaged across animals. Error bars indicate $95 \%$ confidence intervals. The increased evoked responses correlated with the more silent spontaneous activity after spinal cord transection. D, Average rMUA responses evoked in the hindpaw cortex by high-intensity forepaw stimuli after spinal transection in a representative animal that displayed both short-latency responses and long-latency activations. The signal around time 0 was truncated to eliminate the stimulus artifact. $\boldsymbol{E}$, Example of LFP recordings throughout two consecutive high-intensity forepaw stimuli (interstimulus interval $2 \mathrm{~s}$ ) before (left) and after (right) spinal cord transection. During slow-wave activity, high-intensity forepaw stimuli triggered active states in the forepaw cortex that propagated to the hindpaw cortex, generating long-latency activations (arrows).

transection, $S=-0.168$ ) than for low-intensity stimuli (before transection, $S=-0.056$; after transection, $S=-0.067$ ) (Fig. $3 C)$, because low-intensity stimuli are less likely to trigger active states associated with the response than high-intensity stimuli (Hasenstaub et al., 2007; Reig and Sanchez-Vives, 2007). This could explain why the increased forepaw responses were specifically observed with high-intensity stimuli: after the deafferentation imposed by thoracic transection of the spinal cord, forepaw stimuli are more likely to occur when the cortex is more silent, but only high-intensity stimuli are able to consistently trigger active states, and thus to evoke larger cortical responses.

In the cortical state of slow-wave activity induced by spinal cord transection, we expected the active states triggered by highintensity forepaw stimuli to propagate from the forepaw cortex to the hindpaw cortex. We therefore investigated the activity of the hindpaw cortex in response to forepaw stimuli, as evaluated by averaging the rMUA evoked by high-intensity forepaw stimuli, in animals with good MUA recordings from the hindpaw cortex 
A

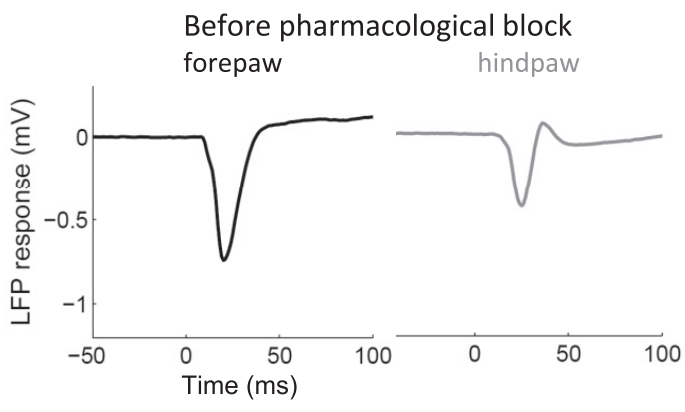

B Before pharmacological block

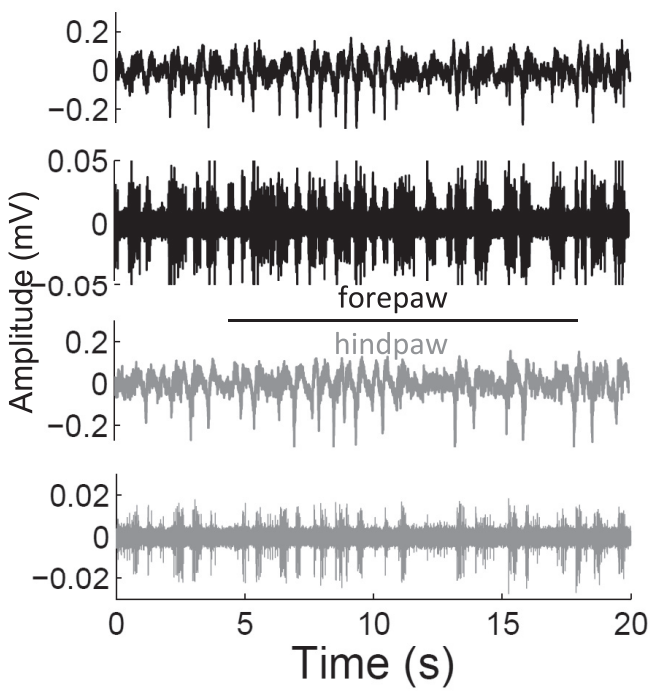

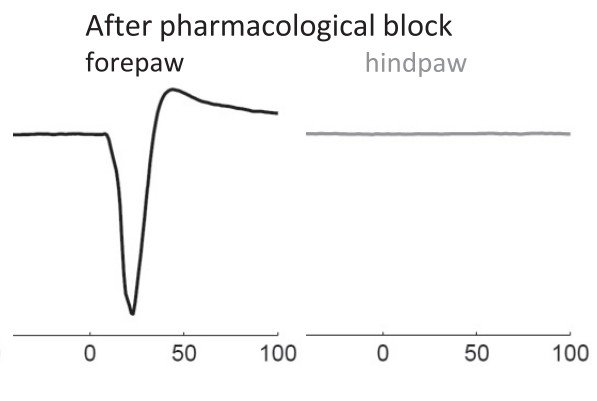

After pharmacological block


D

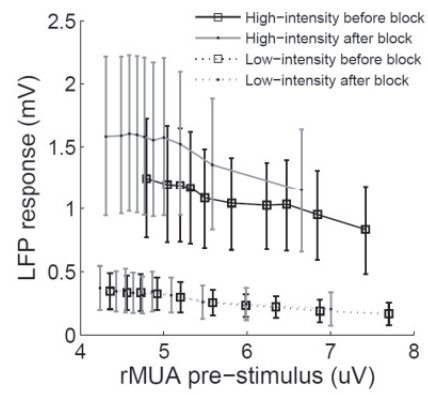

C
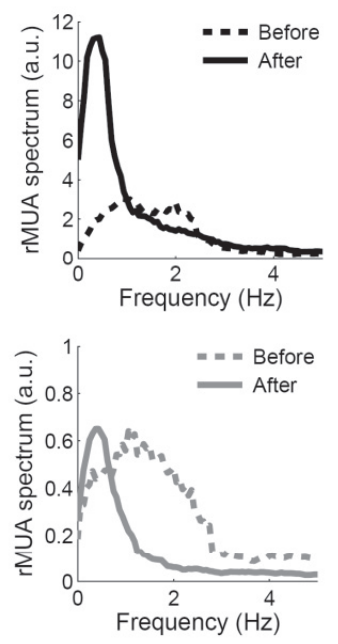

Figure 4. Cortical changes after pharmacological block of the spinal cord. $A$, Changes of cortical responses in a representative animal (as in Fig. $1 A$ ). B, C, Changes in cortical spontaneous activity in a representative animal (as in Fig. 2A, B). D, Relation between cortical spontaneous activity and evoked responses in all animals $(n=8)$ (as in Fig. 3C). The cortical changes observed after pharmacological block of the spinal cord were very similar to the ones observed after spinal cord transection.

$(n=12)$. We observed two types of "responses": (1) shortlatency responses $(<30 \mathrm{~ms})$ and (2) long-latency activations (>50 ms) (Fig. 3D). Short-latency responses were typical somatosensory responses - of the same type as the "non-homologous responses" we described in Moxon et al. (2008)-and were observed in 7 of 12 animals (58\%) before transection and in 7 of 12 animals (58\%) after transection. Long-latency activations were instead due to active states triggered in the forepaw cortex, propagating to the hindpaw cortex, and were observed in 5 of 12 animals (42\%) before transection and in 9 of 12 animals (75\%) after transection. In the animals in which the cortex started from a faster oscillatory state, before spinal cord transection forepaw stimuli did not trigger cortical active states (because the cortex was already active); conversely, during slow-wave activity after spinal cord transection forepaw stimuli did trigger active states in the forepaw cortex, which propagated to the hindpaw cortex and generated the long-latency activations (Fig. $3 E$ ). The increased probability of observing long-latency activations in the hindpaw cortex in response to forepaw stimuli immediately after thoracic spinal cord transection, therefore, is another consequence of the cortical state change induced by the spinal injury.

\section{Pharmacological block of the spinal cord}

To verify that the slower/more silent cortical spontaneous activity and the consequent increased forepaw responses were specifically due to the deafferentation, we performed a set of experiments in which spinal cord conduction was transiently blocked using either lidocaine or TTX (Fig. 4). These experiments were particularly challenging because of the difficulty of locally diffusing the drug without lesioning the spinal cord. Only rats in which the pharmacological block was complete and reversible were included in the study $(n=8)$.

The results we obtained with the pharmacological block were very similar to the ones obtained with the transection (Table 1): hindpaw responses were abolished, confirming the effectiveness of the pharmacological block, but the LFP responses evoked in the forepaw cortex by forepaw stimuli markedly increased (Fig. $4 A$ ) (two-way ANOVA, predrug-postdrug factor: $p=0.0069$; $n=8$ ), particularly in response to high-intensity stimuli (interaction: $p=0.0127$; Tukey: $p=0.0040$ ), but not in response to low-intensity stimuli (Tukey: $p=0.99$ ). In the same way, the spontaneous alternation between active and silent states (hindpaw: $1.05 \pm 0.57 \mathrm{~Hz}$; forepaw: $0.92 \pm 0.56 \mathrm{~Hz}$ ) was significantly slower after the pharmacological block (hindpaw: $0.73 \pm 0.37$ $\mathrm{Hz}$; forepaw: $0.71 \pm 0.34 \mathrm{~Hz}$ ), as revealed by the peak of the rectified MUA spectrum (Fig. $4 B, C$ ) (two-way ANOVA; predrug-postdrug factor: $p=0.0137$; hindpaw-forepaw factor: $p=$ 0.75 ; interaction: $p=0.54 ; n=7)$, and the overall level of cortical spontaneous activity was significantly more silent after the pharmacological block, as revealed by the decreased mean amplitude 
of the rectified MUA (two-way ANOVA; prelesion-postlesion factor: $p=0.0140$; hindpaw-forepaw factor: $p=0.58$; interaction: $p=0.34 ; n=7)$. These changes in spontaneous activity correlated with the evoked responses similarly to the experiments with spinal cord transection (Fig. 4D).

As an additional control condition, we performed a set of experiments in which animals underwent the same experimental protocol, but no lesion or pharmacological block of the spinal cord was performed $(n=9)$. Cortical responses and spontaneous activity were thus evaluated before and after "sham." The amplitude of LFP responses evoked in the forepaw cortex by forepaw stimuli did not change after sham (two-way ANOVA, preshampostsham factor: $p=0.92 ; n=9)$. In same way, the peak of the rectified MUA spectrum did not change after sham (two-way ANOVA, presham-postsham factor: $p=0.40 ; n=7$ ), nor was a decrease of the rectified MUA amplitude observed (two-way ANOVA, presham-postsham factor: $p=0.72 ; n=7)$. Therefore, the results observed after spinal transection are unlikely to be related to unspecific alterations induced by the experimental procedures and are most likely due to the deafferentation.

\section{Discussion}

The main result of the present work is that a complete thoracic transection of the spinal cord produces an immediate functional reorganization in the primary somatosensory cortex of anesthetized rats. While cortical responses evoked by stimuli delivered below the level of the lesion are obviously abolished after spinal transection, cortical responses evoked by stimuli delivered above the level of the lesion markedly increase. Importantly, these increased responses are paralleled by-and correlate with-a slower and overall more silent cortical spontaneous activity, representing a switch to a network state of slow-wave activity. The same immediate cortical changes are observed after reversible pharmacological block of spinal cord conduction, but not after sham. The deafferentation produced by spinal cord injury can thus immediately change the state of large cortical networks, leading to increased cortical responses to stimuli delivered above the level of the lesion.

\section{Changes of cortical responses}

The increased cortical responses to forepaw stimuli we observed immediately after thoracic spinal cord injury are in good agreement with the long-term changes observed in previous studies with functional imaging in rats, using either thoracic contusion (Hofstetter et al., 2003), thoracic transection (Endo et al., 2007), or thoracic bilateral dorsal section of the spinal cord (Ghosh et al., 2010). In particular, using functional magnetic resonance imaging (fMRI) in the same spinal cord injury model that we used, Endo et al. (2007) found increases of blood oxygenation leveldependent (BOLD) signals in the primary somatosensory cortex in response to stimulation of the intact forelimb in the first fMRI protocol they performed $3 \mathrm{~d}$ after injury. Similarly increased cortical responses to forepaw stimuli were reported 1 week after spinal cord injury by Ghosh et al. (2010), using both fMRI and voltage-sensitive dye imaging (VSD). Since BOLD, VSD, and LFP responses to sensory stimuli are reasonably related (Logothetis et al., 2001; Arthurs and Boniface, 2003; Petersen et al., 2003; Goloshevsky et al., 2008), our findings likely represent the early electrophysiological counterpart of the results of Endo et al. (2007) and Ghosh et al. (2010). Importantly, we observed increased cortical responses after spinal transection only with highintensity stimuli, likely activating both the dorsal columns and spinothalamic tract (Lilja et al., 2006), but not with low-intensity stimuli, likely activating only the dorsal columns (Lilja et al., 2006). This difference might help reconciling the studies by Hofstetter et al. (2003), Endo et al. (2007), and Ghosh et al. (2010), who observed long-term cortical reorganization using relatively high-intensity electrical stimuli, with the study by Jain et al. (1995), who reported absence of any cortical reorganization with light tactile stimuli after dorsal column section. Although our experimental approach does not allow us to assess changes in the spatial extent of cortical activation, our results show that increased cortical responses to stimuli above the level of the lesion can be observed immediately after transection of the spinal cord.

\section{Changes of cortical spontaneous activity}

Cortical responses to sensory stimuli highly depend on the ongoing spontaneous activity of cortical networks (Arieli et al., 1996; Wörgötter et al., 1998; Azouz and Gray, 1999; Kisley and Gerstein, 1999; Tsodyks et al., 1999; Kenet et al., 2003; Lakatos et al., 2008). However, virtually all studies investigating the reorganization of cortical responses after deafferentation by peripheral or central injuries-including the studies by Endo et al. (2007) and by Ghosh et al. (2010)_neglect its possible dependence on changes in cortical spontaneous activity. Here we show that immediately after thoracic transection of the spinal cord, cortical spontaneous activity becomes strikingly slower and overall more silent, switching to a state of slow-wave activity similar to that observed during slow-wave sleep (Steriade et al., 1993; Vyazovskiy et al., 2009; Crunelli and Hughes, 2010). The most intuitive explanation for these immediate changes in cortical spontaneous activity is that spinal cord transection likely decreases the tonic inputs to supraspinal somatosensory structures, ultimately producing a cortical effect that resembles a direct thalamocortical deafferentation (Rigas and Castro-Alamancos, 2007; Hirata and CastroAlamancos, 2010). At first glance, it might thus appear surprising that the slowing/silencing occurred not only in the deafferented hindpaw cortex, but also in the supposedly intact forepaw cortex. In the rat, however, the forepaw cortex and the hindpaw cortex are functionally connected (Moxon et al., 2008; Ghosh et al., 2009, 2010), and the physiologic alternation between active and silent states occurs synchronously across large cortical regions (Fox and Armstrong-James, 1986; Steriade, 2006; Mohajerani et al., 2010; see also supplemental Results). Therefore, if one region (e.g., the hindpaw cortex) slows down, adjacent regions (e.g., the forepaw cortex) are also likely to slow down due to physiological synchronization mechanisms (Amzica and Steriade, 1995). Besides deafferentation of somatosensory structures, it is possible that spinal transection also directly affected the activity of brainstem structures and thalamic nuclei regulating cortical synchrony and arousal, contributing to the observed changes in cortical spontaneous activity (Moruzzi and Magoun, 1949; Lindvall et al., 1974; Hobson et al., 1975; Foote et al., 1980; Aston-Jones and Bloom, 1981a,b; Fox and Armstrong-James, 1986; Satoh and Fibiger, 1986; Hallanger et al., 1987; Steriade et al., 1990; Aguilar and Castro-Alamancos, 2005; Ren et al., 2009). Importantly, slower cortical activity after spinal cord injury has been previously observed with EEG recordings in patients (Tran et al., 2004; Boord et al., 2008), and both slower cortical activity (Wydenkeller et al., 2009) and long-term cortical reorganization (Wrigley et al., 2009) correlate with the emergence of neuropathic pain. From a translational perspective, it is thus tempting to suggest-with all the necessary caveats of comparing data from awake patients and anesthetized rats - that the immediate slowing of cortical spontaneous activity after spinal cord injury described here might 
have a pathophysiological role for long-term cortical reorganization and neuropathic pain.

\section{Relation between cortical spontaneous activity and evoked responses}

The immediate cortical reorganization observed after peripheral injuries is classically explained in terms of unmasking of latent excitatory synapses by removal of inhibition, which could occur at cortical level (Jacobs and Donoghue, 1991), in the thalamus (Nicolelis et al., 1993; Faggin et al., 1997), and possibly also at lower levels. Our increased forepaw responses immediately after thoracic transection of the spinal cord could indeed be explained, at least in part, by classical unmasking. However, our results show that the increased cortical responses are directly related to the slower/more silent cortical spontaneous activity, thus offering an additional mechanism to explain immediate changes in cortical responses after deafferentation. In the rat somatosensory system, brief peripheral stimuli evoke larger cortical responses when the cortex is in a silent compared to an active state (Petersen et al., 2003; Sachdev et al., 2004), particularly when the stimuli themselves are able to trigger active states associated with the responses (Hasenstaub et al., 2007; Reig and Sanchez-Vives, 2007). This inverse relation between the level of spontaneous activity and the amplitude of evoked responses is clearly present in our data. The lesion itself transitions the somatosensory cortex to a slower/ more silent state, so that forepaw stimuli delivered after spinal transection are more likely to occur when the cortex is more silent compared to before the lesion, and consequently evoke larger cortical responses associated with active states that propagate from the forepaw cortex to the hindpaw cortex. The mechanism underlying this phenomenon is essentially the same by which cortical somatosensory responses are enhanced in quiescent states compared to attentive states in awake animals (CastroAlamancos, 2004). Our results thus go beyond the classical idea of unmasking identified with peripheral injuries, revealing that changes in the state of the brain could play a critical role in the early mechanisms of cortical reorganization after deafferentation.

\section{Pharmacological block of the spinal cord}

It is important to remark that we observed the same immediate cortical changes after reversible pharmacological block of the spinal cord, and that no changes were observed after sham. Therefore, these cortical changes are unlikely to be due to the axotomy of efferent corticospinal cells (Hains et al., 2003; Lee et al., 2004; Jurkiewicz et al., 2006), to unspecific systemic reactions to the spinal injury, or to the experimental protocol, but are most likely specifically due to the deafferentation. Our result that deafferentation affects cortical slow-wave activity supports the view that this important sleep rhythm is not purely cortical, as usually considered, but is also critically controlled by subcortical structures (Fox and Armstrong-James, 1986; Manjarrez et al., 2002; Crunelli and Hughes, 2010). The possible mechanistic role played by slow-wave activity in shaping long-term cortical reorganization after deafferentation (Aton et al., 2009) should be further investigated in future studies.

\section{Conclusions}

In conclusion, the deafferentation due to spinal cord injury can immediately change the state of large cortical networks, and this change in the state of the brain plays a critical role in the immediate functional reorganization of the primary somatosensory cortex after spinal cord injury. More in general, our study sug- gests that the state of the spinal cord and the state of the brain are tightly connected.

\section{References}

Aguilar JR, Castro-Alamancos MA (2005) Spatiotemporal gating of sensory inputs in thalamus during quiescent and activated states. J Neurosci 25:10990-11002.

Aguilar J, Morales-Botello ML, Foffani G (2008) Tactile responses of hindpaw, forepaw and whisker neurons in the thalamic ventrobasal complex of anesthetized rats. Eur J Neurosci 27:378-387.

Amzica F, Steriade M (1995) Disconnection of intracortical synaptic linkages disrupts synchronization of a slow oscillation. J Neurosci 15:4658-4677.

Arieli A, Sterkin A, Grinvald A, Aertsen A (1996) Dynamics of ongoing activity: explanation of the large variability in evoked cortical responses. Science 273:1868-1871.

Arthurs OJ, Boniface SJ (2003) What aspect of the fMRI BOLD signal best reflects the underlying electrophysiology in human somatosensory cortex? Clin Neurophysiol 114:1203-1209.

Aston-Jones G, Bloom FE (1981a) Activity of norepinephrine-containing locus coeruleus neurons in behaving rats anticipates fluctuations in the sleep-waking cycle. J Neurosci 1:876-886.

Aston-Jones G, Bloom FE (1981b) Norepinephrine-containing locus coeruleus neurons in behaving rats exhibit pronounced responses to nonnoxious environmental stimuli. J Neurosci 1:887-900.

Aton SJ, Seibt J, Dumoulin M, Jha SK, Steinmetz N, Coleman T, Naidoo N, Frank MG (2009) Mechanisms of sleep-dependent consolidation of cortical plasticity. Neuron 61:454-466.

Azouz R, Gray CM (1999) Cellular mechanisms contributing to response variability of cortical neurons in vivo. J Neurosci 19:2209-2223.

Boord P, Siddall PJ, Tran Y, Herbert D, Middleton J, Craig A (2008) Electroencephalographic slowing and reduced reactivity in neuropathic pain following spinal cord injury. Spinal Cord 46:118-123.

Bruehlmeier M, Dietz V, Leenders KL, Roelcke U, Missimer J, Curt A (1998) How does the human brain deal with a spinal cord injury? Eur J Neurosci 10:3918-3922.

Byrne JA, Calford MB (1991) Short-term expansion of receptive fields in rat primary somatosensory cortex after hindpaw digit denervation. Brain Res 565:218-224.

Calford MB, Tweedale R (1988) Immediate and chronic changes in responses of somatosensory cortex in adult flying-fox after digit amputation. Nature 332:446-448.

Calford MB, Tweedale R (1991a) Acute changes in cutaneous receptive fields in primary somatosensory cortex after digit denervation in adult flying fox. J Neurophysiol 65:178-187.

Calford MB, Tweedale R (1991b) Immediate expansion of receptive fields of neurons in area $3 \mathrm{~b}$ of macaque monkeys after digit denervation. Somatosens Mot Res 8:249-260.

Castro-Alamancos MA (2000) Origin of synchronized oscillations induced by neocortical disinhibition in vivo. J Neurosci 20:9195-9206.

Castro-Alamancos MA (2004) Absence of rapid sensory adaptation in neocortex during information processing states. Neuron 41:455-464.

Castro-Alamancos MA, Rigas P (2002) Synchronized oscillations caused by disinhibition in rodent neocortex are generated by recurrent synaptic activity mediated by AMPA receptors. J Physiol 542:567-581.

Chapin JK, Lin CS (1984) Mapping the body representation in the SI cortex of anesthetized and awake rats. J Comp Neurol 229:199-213.

Crunelli V, Hughes SW (2010) The slow $(<1 \mathrm{~Hz})$ rhythm of non-REM sleep: a dialogue between three cardinal oscillators. Nat Neurosci 13:9-17.

Curt A, Alkadhi H, Crelier GR, Boendermaker SH, Hepp-Reymond MC, Kollias SS (2002) Changes of non-affected upper limb cortical representation in paraplegic patients as assessed by fMRI. Brain 125:2567-2578.

Cusick CG, Wall JT, Whiting JH Jr, Wiley RG (1990) Temporal progression of cortical reorganization following nerve injury. Brain Res 537:355-358.

Doetsch GS, Harrison TA, MacDonald AC, Litaker MS (1996) Short-term plasticity in primary somatosensory cortex of the rat: rapid changes in magnitudes and latencies of neuronal responses following digit denervation. Exp Brain Res 112:505-512.

Endo T, Spenger C, Tominaga T, Brené S, Olson L (2007) Cortical sensory map rearrangement after spinal cord injury: fMRI responses linked to Nogo signalling. Brain 130:2951-2961. 
Faggin BM, Nguyen KT, Nicolelis MA (1997) Immediate and simultaneous sensory reorganization at cortical and subcortical levels of the somatosensory system. Proc Natl Acad Sci U S A 94:9428-9433.

Foffani G, Morales-Botello ML, Aguilar J (2009) Spike timing, spike count, and temporal information for the discrimination of tactile stimuli in the rat ventrobasal complex. J Neurosci 29:5964-5973.

Foote SL, Aston-Jones G, Bloom FE (1980) Impulse activity of locus coeruleus neurons in awake rats and monkeys is a function of sensory stimulation and arousal. Proc Natl Acad Sci U S A 77:3033-3037.

Fox K, Armstrong-James M (1986) The role of the anterior intralaminar nuclei and N-methyl D-aspartate receptors in the generation of spontaneous bursts in rat neocortical neurones. Exp Brain Res 63:505-518.

Ghosh A, Sydekum E, Haiss F, Peduzzi S, Zörner B, Schneider R, Baltes C, Rudin M, Weber B, Schwab ME (2009) Functional and anatomical reorganization of the sensory-motor cortex after incomplete spinal cord injury in adult rats. J Neurosci 29:12210-12219.

Ghosh A, Haiss F, Sydekum E, Schneider R, Gullo M, Wyss MT, Mueggler T, Baltes C, Rudin M, Weber B, Schwab ME (2010) Rewiring of hindlimb corticospinal neurons after spinal cord injury. Nat Neurosci 13:97-104.

Goloshevsky AG, Silva AC, Dodd SJ, Koretsky AP (2008) BOLD fMRI and somatosensory evoked potentials are well correlated over a broad range of frequency content of somatosensory stimulation of the rat forepaw. Brain Res 1195:67-76.

Green JB, Sora E, Bialy Y, Ricamato A, Thatcher RW (1998) Cortical sensorimotor reorganization after spinal cord injury: an electroencephalographic study. Neurology 50:1115-1121.

Hains BC, Black JA, Waxman SG (2003) Primary cortical motor neurons undergo apoptosis after axotomizing spinal cord injury. J Comp Neurol 462:328-341.

Hallanger AE, Levey AI, Lee HJ, Rye DB, Wainer BH (1987) The origins of cholinergic and other subcortical afferents to the thalamus in the rat. J Comp Neurol 262:105-124.

Hasenstaub A, Sachdev RN, McCormick DA (2007) State changes rapidly modulate cortical neuronal responsiveness. J Neurosci 27:9607-9622.

Hirata A, Castro-Alamancos MA (2010) Neocortex network activation and deactivation states controlled by the thalamus. J Neurophysiol 103:1147-1157.

Hobson JA, McCarley RW, Wyzinski PW (1975) Sleep cycle oscillation: reciprocal discharge by two brainstem neuronal groups. Science 189:55-58.

Hofstetter CP, Schweinhardt P, Klason T, Olson L, Spenger C (2003) Numb rats walk - a behavioural and fMRI comparison of mild and moderate spinal cord injury. Eur J Neurosci 18:3061-3068.

Jacobs KM, Donoghue JP (1991) Reshaping the cortical motor map by unmasking latent intracortical connections. Science 251:944-947.

Jain N, Florence SL, Kaas JH (1995) Limits on plasticity in somatosensory cortex of adult rats: hindlimb cortex is not reactivated after dorsal column section. J Neurophysiol 73:1537-1546.

Jain N, Catania KC, Kaas JH (1997) Deactivation and reactivation of somatosensory cortex after dorsal spinal cord injury. Nature 386:495-498.

Jain N, Qi HX, Collins CE, Kaas JH (2008) Large-scale reorganization in the somatosensory cortex and thalamus after sensory loss in macaque monkeys. J Neurosci 28:11042-11060.

Jurkiewicz MT, Crawley AP, Verrier MC, Fehlings MG, Mikulis DJ (2006) Somatosensory cortical atrophy after spinal cord injury: a voxel-based morphometry study. Neurology 66:762-764.

Kaas JH, Qi HX, Burish MJ, Gharbawie OA, Onifer SM, Massey JM (2008) Cortical and subcortical plasticity in the brains of humans, primates, and rats after damage to sensory afferents in the dorsal columns of the spinal cord. Exp Neurol 209:407-416.

Kao T, Shumsky JS, Murray M, Moxon KA (2009) Exercise induces cortical plasticity after neonatal spinal cord injury in the rat. J Neurosci 29:7549-7557.

Kelahan AM, Doetsch GS (1984) Time-dependent changes in the functional organization of somatosensory cerebral cortex following digit amputation in adult raccoons. Somatosens Res 2:49-81.

Kenet T, Bibitchkov D, Tsodyks M, Grinvald A, Arieli A (2003) Spontaneously emerging cortical representations of visual attributes. Nature 425:954-956.

Kisley MA, Gerstein GL (1999) Trial-to-trial variability and statedependent modulation of auditory-evoked responses in cortex. J Neurosci 19:10451-10460.

Lakatos P, Karmos G, Mehta AD, Ulbert I, Schroeder CE (2008) Entrain- ment of neuronal oscillations as a mechanism of attentional selection. Science 320:110-113.

Lee BH, Lee KH, Kim UJ, Yoon DH, Sohn JH, Choi SS, Yi IG, Park YG (2004) Injury in the spinal cord may produce cell death in the brain. Brain Res 1020:37-44.

Lilja J, Endo T, Hofstetter C, Westman E, Young J, Olson L, Spenger C (2006) Blood oxygenation level-dependent visualization of synaptic relay stations of sensory pathways along the neuroaxis in response to graded sensory stimulation of a limb. J Neurosci 26:6330-6336.

Lindvall O, Björklund A, Nobin A, Stenevi U (1974) The adrenergic innervation of the rat thalamus as revealed by the glyoxylic acid fluorescence method. J Comp Neurol 154:317-347.

Logothetis NK, Pauls J, Augath M, Trinath T, Oeltermann A (2001) Neurophysiological investigation of the basis of the fMRI signal. Nature 412:150-157.

Manjarrez E, Rojas-Piloni G, Vazquez D, Flores A (2002) Cortical neuronal ensembles driven by dorsal horn spinal neurones with spontaneous activity in the cat. Neurosci Lett 318:145-148.

Merzenich MM, Kaas JH, Wall JT, Sur M, Nelson RJ, Felleman DJ (1983) Progression of change following median nerve section in the cortical representation of the hand in areas $3 \mathrm{~b}$ and 1 in adult owl and squirrel monkeys. Neuroscience 10:639-665.

Mohajerani MH, McVea DA, Fingas M, Murphy TH (2010) Mirrored bilateral slow-wave cortical activity within local circuits revealed by fast bihemispheric voltage-sensitive dye imaging in anesthetized and awake mice. J Neurosci 30:3745-3751.

Moore CI, Stern CE, Dunbar C, Kostyk SK, Gehi A, Corkin S (2000) Referred phantom sensations and cortical reorganization after spinal cord injury in humans. Proc Natl Acad Sci U S A 97:14703-14708.

Moruzzi G, Magoun HW (1949) Brain stem reticular formation and activation of the EEG. Electroencephalogr Clin Neurophysiol 1:455-473.

Moxon KA, Hale LL, Aguilar J, Foffani G (2008) Responses of infragranular neurons in the rat primary somatosensory cortex to forepaw and hindpaw tactile stimuli. Neuroscience 156:1083-1092.

Nicolelis MA, Lin RC, Woodward DJ, Chapin JK (1993) Induction of immediate spatiotemporal changes in thalamic networks by peripheral block of ascending cutaneous information. Nature 361:533-536.

Paxinos G, Watson C (1986) The rat brain in stereotaxic coordinates, Ed 2. Sydney: Academic.

Petersen CC, Hahn TT, Mehta M, Grinvald A, Sakmann B (2003) Interaction of sensory responses with spontaneous depolarization in layer $2 / 3$ barrel cortex. Proc Natl Acad Sci U S A 100:13638-13643.

Reig R, Sanchez-Vives MV (2007) Synaptic transmission and plasticity in an active cortical network. PLoS One 2:e670.

Ren Y, Zhang L, Lu Y, Yang H, Westlund KN (2009) Central lateral thalamic neurons receive noxious visceral mechanical and chemical input in rats. J Neurophysiol 102:244-258.

Rigas P, Castro-Alamancos MA (2007) Thalamocortical up states: differential effects of intrinsic and extrinsic cortical inputs on persistent activity. J Neurosci 27:4261-4272.

Sachdev RN, Ebner FF, Wilson CJ (2004) Effect of subthreshold up and down states on the whisker-evoked response in somatosensory cortex. J Neurophysiol 92:3511-3521.

Sakata S, Harris KD (2009) Laminar structure of spontaneous and sensoryevoked population activity in auditory cortex. Neuron 64:404-418.

Sanchez-Vives MV, McCormick DA (2000) Cellular and network mechanisms of rhythmic recurrent activity in neocortex. Nat Neurosci 3:1027-1034.

Satoh K, Fibiger HC (1986) Cholinergic neurons of the laterodorsal tegmental nucleus: efferent and afferent connections. J Comp Neurol 253:277-302.

Steriade M (2006) Grouping of brain rhythms in corticothalamic systems. Neuroscience 137:1087-1106.

Steriade M, Datta S, Paré D, Oakson G, Curró Dossi RC (1990) Neuronal activities in brain-stem cholinergic nuclei related to tonic activation processes in thalamocortical systems. J Neurosci 10:2541-2559.

Steriade M, Nuñez A, Amzica F (1993) A novel slow ( $<1 \mathrm{~Hz}$ ) oscillation of neocortical neurons in vivo: depolarizing and hyperpolarizing components. J Neurosci 13:3252-3265.

Tandon S, Kambi N, Lazar L, Mohammed H, Jain N (2009) Large-scale expansion of the face representation in somatosensory areas of the 
lateral sulcus after spinal cord injuries in monkeys. J Neurosci 29:12009-12019.

Tran Y, Boord P, Middleton J, Craig A (2004) Levels of brain wave activity $(8-13 \mathrm{~Hz})$ in persons with spinal cord injury. Spinal Cord 42:73-79.

Tsodyks M, Kenet T, Grinvald A, Arieli A (1999) Linking spontaneous activity of single cortical neurons and the underlying functional architecture. Science 286:1943-1946.

Turnbull BG, Rasmusson DD (1990) Acute effects of total or partial digit denervation on raccoon somatosensory cortex. Somatosens Mot Res 7:365-389.

Vyazovskiy VV, Olcese U, Lazimy YM, Faraguna U, Esser SK, Williams JC, Cirelli C, Tononi G (2009) Cortical firing and sleep homeostasis. Neuron 63:865-878.

Wall JT, Cusick CG (1984) Cutaneous responsiveness in primary somato- sensory (S-I) hindpaw cortex before and after partial hindpaw deafferentation in adult rats. J Neurosci 4:1499-1515.

Wall PD, Egger MD (1971) Formation of new connexions in adult rat brains after partial deafferentation. Nature 232:542-545.

Wörgötter F, Suder K, Zhao Y, Kerscher N, Eysel UT, Funke K (1998) Statedependent receptive-field restructuring in the visual cortex. Nature 396:165-168.

Wrigley PJ, Press SR, Gustin SM, Macefield VG, Gandevia SC, Cousins MJ, Middleton JW, Henderson LA, Siddall PJ (2009) Neuropathic pain and primary somatosensory cortex reorganization following spinal cord injury. Pain 141:52-59.

Wydenkeller S, Maurizio S, Dietz V, Halder P (2009) Neuropathic pain in spinal cord injury: significance of clinical and electrophysiological measures. Eur J Neurosci 30:91-99. 